\title{
Optimal One-Max Strategy with Dynamic Island Models
}

\author{
Adrien Goëffon \\ LERIA - University of Angers \\ France \\ adrien.goeffon@univ-angers.fr
}

\author{
Frédéric Lardeux \\ LERIA - University of Angers \\ France \\ frederic.lardeux@univ-angers.fr
}

\begin{abstract}
In this paper, we recall the dynamic island model concept, in order to dynamically select local search operators within a multi-operator genetic algorithm. We use a fullyconnected island model, where each island is assigned to a local search operator. Selection of operators is simulated by migration steps, whose policies depend on a learning process. The efficiency of this approach is assessed in comparing, for the One-Max Problem, theoretical and ideal results to those obtained by the model. Experiments show that the model has the expected behavior and is able to regain the optimal local search strategy for this well-known problem.
\end{abstract}

Keywords-evolutionary computation; local search; island models; autonomous search; operator selection;

\section{INTRODUCTION}

Evolutionary algorithms are widely used for tackling NPhard problems [1], [2], [3]. Although their efficiency on numerous optimization problem is well-established, their performance and robustness depend on various elements like the problem modeling, the search space properties or global parametrizations [4]. These technical and execution alternatives make the ad hoc resolution of combinatorial optimization problems difficult to tackle by non-specialist users. Recent approaches use autonomous algorithms [5], which are able to analyze their own performances in order to auto-adapt their behavior in function of the problem to solve.

Island Models [6] are simultaneously considering a set of populations clustered in islands which are evolving independently during some search stages while interacting periodically. This model, which constitutes an additional abstraction level in comparison to classical genetic and memetic algorithms, allows to propose several diversification levels and to simplify its parallelization.

Most of the time, island models are used in a static way, where individuals are migrating from population to population following a determinate scheme [7], or are specifically chosen in order to reinforce the populations diversities [8], [9], [10]. Nevertheless, it is possible to dynamically regulate migrations between islands in considering a transition matrix [11]. Such a model can reinforce or reduce the migration probabilities during the evolutionary process in function of the impact of previous analog migrations. The aim is to autoadapt migration without any given scheme, to dynamically regulate the gathering or isolation of individuals in function of the search progress, and consequently to adapt the population sizes.

In classical uniform island models, islands are following the same evolutionary rules, so they differ only by their individuals. The dynamic model allows to regulate interactions between individuals or group of individuals. We propose to extend this model in assigning to each island different local search operators. An appropriate and autonomous regulation of migration flows will affect dynamically the resources to the most pertinent operators in function of the search progress. In experimenting this model without crossovers but with a proper local search operator for each island, the objective is not only to regulate the interactions between individuals, but to simulate a reactive controller which assigns individuals to the most promising islands.

In section 2 and after a brief recall of the dynamic island model used, we present our island-based algorithm which aims at simulating an autonomous local search algorithm, or more precisely a population-based algorithm with an adaptive selection of local search operators. Section 3 presents the One-Max problem and the theoretical optimal strategies to solve it with local search (LS). In section 4, experimentations will show the validity of our approach in confronting theoretical and experimental results.

\section{ISLAND MODELS FRAMEWORK}

\section{A. Island Models as a Complete Digraph}

In [11] we proposed an island model framework which dynamically supervises the commonly-used specification parameters [10] like the number of individuals undergoing migration, the policy for selecting immigrants or the topology of the communication among subpopulations. An island model topology is represented by a complete labeled digraph $G=\left(X, X^{2}\right)$.

For an easier representation, we identify each island by an ordinal; for example, $\left(\{1, \ldots, N\},\{1, \ldots, N\}^{2}\right)$ represents the topology of a $N$-islands model.

Migration policies are given by a transition (stochastic) matrix $T$ of size $n$, where $T(i, j)$ represents the probability for an individual to migrate from island $i$ to island $j$ (or to stay at the same island if $i=j$ ). Since the model is dynamic, $T$ can be updated before of after each migration 
process. One can denote $T_{t}$ the matrix at time (or generation) $t$.

An application of this dynamic evolution of the model topology is to determine pertinent migration probabilities at each time of the search, considering a classical multipopulation based genetic algorithm. The dynamic regulation of migration policies can produce different size islands, which prevents poor-quality subpopulations or islands to require as many computational effort as promising ones. However, if different islands represent different mutation or local search operators, then the aim is to dynamically provide a well-adapted repartition of individuals in function of these operators and considering the search progression, which can be assimilated to an operator selection process.

\section{B. Autonomous Operator Selection}

The aim of this work is to use this dynamic island model to autonomously select local search operators within a classical evolutionary algorithm. In order to assess the relevance of this approach, we will use the model considering a population-based local search algorithm, with no crossover and where each island is associated to a particular local search operator. Here, contrary to recent works [5], the goal is not to forecast the most promising crossovers between individuals like in classical island models, but to detect at each time of the search the most relevant LS operators. This application constitutes an original approach in defining autonomous algorithms.

\section{Migration Policy}

Algorithm 1 is the generic algorithm we used for the autonomous operator selection within an island model context. In order to allow a maximum of adaptability, we chose to update the migration process after each local search iteration (for the whole population). Ideational, less frequent mutations process do not minimize the effective number of mutations (individuals moving to other islands) but only provide a less reactive search. As a dynamic algorithm, transition values are expected to be regulate accordingly.

\section{Initialize population;

Update the Transition Matrix T;

Migration Process;

until stop condition;

Algorithm 1: Generic Dynamic Island Model (DIM) Algorithm.

The crucial point concerns the update of the transition matrix $T$, which follows a learning process:

$$
T_{t}=(1-\beta)\left(\alpha \cdot T_{t-1}+(1-\alpha) \cdot R_{t}\right)+\beta \cdot N_{t}
$$

$R_{t}$ is a reward matrix, computed after migration process $t-1$ and LS step $t$, and which takes into account the comparative pertinence of the last migrations. Using an intensive strategy, for each island, the migration which have brought the best average accuracy score acc of individuals (typically their fitness improvement) receives the maximum reward. More formally, if $M_{i j t}$ is the set of individuals which have migrated from island $i$ to island $j$ in migration process $t-1\left(\cup_{i} M_{i j t}\right.$ is the set of individuals in island $j$ during iteration $t$ ):

$$
\begin{gathered}
R_{t}(i, j)= \begin{cases}1 /|B| & \text { if } j \in B, \\
0 & \text { otherwise, }\end{cases} \\
\text { with } B=\underset{j^{\prime}}{\operatorname{argmax}} \frac{\sum_{x \in M_{i j^{\prime} t}} \operatorname{acc}(x)}{\left|M_{i j^{\prime} t}\right|}
\end{gathered}
$$

$N_{t}$ is a noise stochastic matrix with random values.

The two parameters $\alpha$ and $\beta$ allow to manage the update of the transition matrix. $\alpha$ represents the importance of the knowledge accumulated during the last migrations and $\beta$ the amount of noise which is necessary to explore alternative ways and to keep the model reactive.

\section{ONE-MAX PROBLEM}

The One-Max problem is a simple and well-known problem, commonly used to assess the performance of Adaptive Operator Selection algorithms [12], [13]. The $n$-bits OneMax problem considers $n$-length bit strings; starting from $0^{n}$ individuals (i.e. strings made up of $n$ zeros), the aim is to maximize the number of ones, that is to reach the $1^{n}$ bit string. The score of a bit string $x$, noted $|x|_{1}$, corresponds to its number of ones.

\section{A. One-Max Mutation Operators}

Recent works cited above use four mutation (or local search) operators: bit-flip, which flips every bit with probability $1 / n$, and $k$-flip (with $k=\{1,3,5\}$ ), which flips exactly $k$ bits. In the following and depending on the context, bit-flip and $k$-flip can denote the mutation operator as well as the corresponding neighborhood relation. $k$ flip can easily be modelized as a neighborhood relation $\mathcal{N}_{k}:\{0,1\}^{n} \rightarrow 2^{\{0,1\}^{n}}$ such as $x^{\prime} \in \mathcal{N}_{k}(x)$ if and only if $\left|h\left(x, x^{\prime}\right)\right|=k$ (hamming distance). It is more difficult to exprim the bit-flip operator with a neighborhood relation, since it corresponds to a complete neighborhood with a nonuniform move probability. However, one bit-flip move can be reduced in one $k$-flip move with a determined probability of chosing $k$.

Intuitively, the 5-flip operator mutation will be more efficient when applied on weak individuals (with a majority of zeros), while 1-flip will improve with a higher probability individuals with a high proportion of ones. This point is 
corroborated by the theoretical comparison of operators hereinafter.

\section{B. Most Accurate Operators}

Definition 1: Let $x \in\{0,1\}^{n}$ an individual of score $s$, $M=\left\{\mu_{1}, \ldots, \mu_{l}\right\}$ a mutation operators set and $x_{1}, \ldots, x_{l}$ $l$ neighbors of $x$ such that $x \stackrel{\mu_{k}}{\rightarrow} x_{k}$ ( $x_{k}$ results from the mutation on $x$ by $\left.\mu_{k}\right) . s_{1}, \ldots, s_{l}$ are the respective scores of individuals $x_{1}, \ldots, x_{l}$. We define the domination rate $\chi\left(\mu_{k}, M, x\right)$ of an operator $\mu_{k}$ on $M$ in function of $x$, the probability $p\left(\Delta s\left(x, x_{k}\right)>\Delta s\left(x, x_{k^{\prime}}\right), \forall k^{\prime} \in\{1, \ldots, l\} \backslash\right.$ $\{k\})$, which is equal to:

$$
\sum_{i=1}^{n}\left(p\left(\Delta s\left(x, x_{k}\right)=i\right) \prod_{k^{\prime} \neq k} \sum_{j=0}^{i-1} p\left(\Delta s\left(x, x_{k^{\prime}}\right)=j\right)\right)
$$

For $k$-flip operators $\left(x^{\prime}\right.$, of score $s^{\prime}$, is the transformation of $x$, of score $s$, by $k$-flip), we have :

$$
p_{k}\left(\Delta s\left(x, x^{\prime}\right)=i\right)= \begin{cases}\frac{C_{n-s}^{i} C_{s}^{k-i}}{C_{n}^{k}} & \text { if } i>0 \\ 1-p\left(s^{\prime}>s\right) & \text { if } i=0 .\end{cases}
$$

For bit-flip operator, $p_{\text {bit }}\left(\Delta s\left(x, x^{\prime}\right)=i\right)=$

$$
\sum_{k=1}^{n}\left(C_{n}^{k} \frac{1}{n^{k}}\left(1-\frac{1}{n}\right)^{n-k} p_{k}\left(\Delta s\left(x, x^{\prime}\right)=i\right)\right)
$$

The domination rates evolution of the four considered operators in function of the score of an individual is shown in Figure 1 (with $M=\{1$-flip, 3-flip, 5-flip, bit-flip $\}$ ).

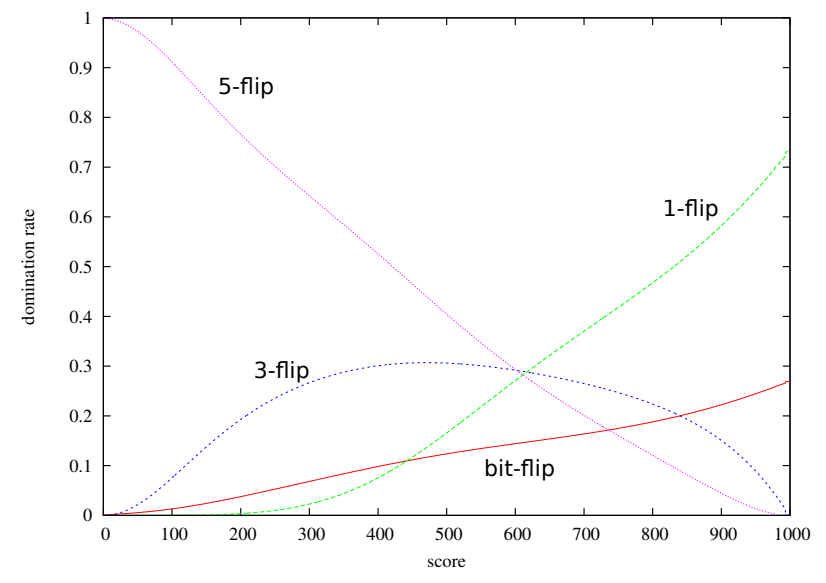

Figure 1. Domination rates evolution for the 1000-bits One-Max problem.

\section{EXPERIMENTATIONS}

In this section we show that the behavior of our population-based local search algorithm is very close of the theoretical results. Moreover, we remark that it is not very dependent of the parameter tuning.

\section{A. Theoretical vs Empirical Results}

As described in section III, our algorithm starts with a population of $0^{n}$ individuals. The expected behavior during the search is to use the 5-flip operator when the population quality is weak (at the beginning), then the 3-flip operator and finally the 1-flip operator when the population quality is sufficiently high. In our experiments, this can be observed by the evolution of the population size in each island with respect to the migrations. The more an island attracts individuals, the more its assigned operator is applied.

Parameters for this experiment are:

- number of islands: 4 (one for each LS operator)

- population size: 400

- initial probabilities of migrations: 1 to stay in the same island

- $\alpha: 0.8$

- $\beta: 0.01$

In addition, let us precise that both fitness function and accuracy score used are the simple bit string score.

To compare the experimental results with the theoretical values, we represent in Figure 2 the population size in each island with respect to the average fitness of the population. The fact that this evolution of population sizes, i.e. the computational effort of each operators, match with the theoretical domination rates, show the accuracy of the proposed model and its pertinence to simulate an operator selection mechanism.

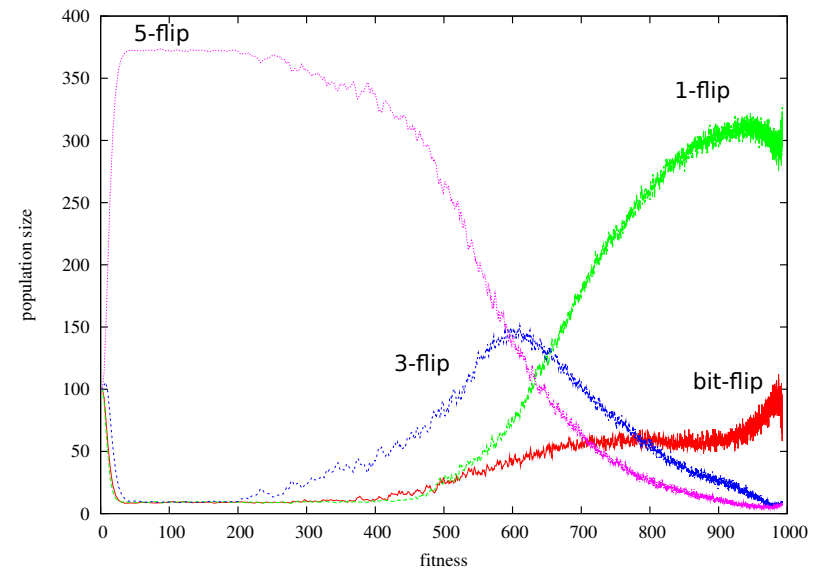

Figure 2. Evolution of the population size in each island with respect to the average fitness of the population.

\section{B. Parametrization}

As presented in section II-C, this dynamic island model requires a few conceptual parameters, the number of individuals, the initial placement of individuals and transition matrix values, as well as the update matrix parameters $\alpha$ and $\beta$. 
1) Population Size: Our experiments show that the behavior of the search is globally identical with a small or a large population (more than 100 individuals by island), even if more individuals make the model a little more robust and less dependent to noise. With a small population, it is necessary to extend the number of steps, but the global number of operations is lower and updating the transition matrix is faster. A population size equal to $100 \times$ number of islands seems to be an ideal trade-off between running time and migrations number if we want to keep a nice matching to the theoretical operator suitability, but in practical use, and since this dynamic model is sufficiently inertial and reactive, smaller population provides equivalent results in terms of solution quality. This point will be assess on realuse problems in future works.

2) Initial Transition Matrix: Except with random values, one can initialize the transition matrix with two natural strategies: first, with a fully partitioned island model (identity matrix); secondly, with a fully connected one (same value everywhere), which makes to each individual the possibility to be affect by any combination of operators in the first steps. Both possibilities have been tested, with no impact on the results; after a few migration steps, both behaviors become identical thanks to the reactive characteristics of the model (on condition that $\beta \neq 0$, see section IV-B3).

3) Dynamic Model Parameters: Default used values for $\alpha$ and $\beta$ are respectively 0.8 and 0.01 . An increasing value of $\alpha$ makes the search slower since informations obtained by recent migrations are less considered for the update. On the contrary, decreasing value of $\alpha$ minimizes the impact of the knowledge (learning process) and overestimates the last migration effects, so the search can be wrong oriented by a migration which provides exceptionally a good result.

The influence of $\beta$ is important, but its exact setting is not crucial to the smooth-running of the algorithm, even if a too high value of $\beta$ make the search slower. On the other hand, it must make sure that $\beta \neq 0$, otherwise some islands can become and stay unreachable (transition probability equal to $0)$.

\section{CONCLUSION}

This paper presents an original and efficient approach to design an autonomous local search algorithm with an accurate selection of operators. The proposed mechanism use a dynamic island model, where each island represents an operator. A learning process regulates and adapts migration policies during the search depending to the impact of previous migrations. At each stage of the search, the more efficient operators receive dynamically the great majority of computational resources. In other words, the model is able to auto-adapt the attractive power of each islands.

This application is an extension of the dynamic island model approach. In previous work, we focus on the capacity for the model to dynamically regulate the interaction between individuals in an evolutionary context, with crossovers and the same configuration on each island, with promising results. Here, we dissociate the exploitation / exploration dilemma to focus on the capacity to allocate with relevance the resources to the most suitable operators. For that, we used an experimental protocol which makes possible to assess the real efficiency of the model (One-Max problem and comparison with theoretical values). The next step is to apply this operator selection strategy to difficult problems, and then to assemble this heterogeneous model within a more general evolutionary context.

\section{REFERENCES}

[1] Z. Michalewicz, Genetic algorithms + data structures = evolution programs (3rd ed.). London, UK: Springer-Verlag, 1996.

[2] A. Eiben and J. Smith, Introduction to Evolutionary Computing, ser. Natural Computing Series. Springer, 2003.

[3] K. De Jong, Evolutionary computation: a unified approach. MIT Press, 2006.

[4] F. G. Lobo, C. F. Lima, and Z. Michalewicz, Eds., Parameter Setting in Evolutionary Algorithms, ser. Studies in Computational Intelligence. Springer, 2007, vol. 54.

[5] J. Maturana, F. Lardeux, and F. Saubion, "Autonomous operator management for evolutionary algorithms," J. Heuristics, vol. 16, no. 6, pp. 881-909, 2010.

[6] D. Whitley, S. Rana, and R. B. Heckendorn, "The island model genetic algorithm: On separability, population size and convergence," Journal of Computing and Information Technology, vol. 7, pp. 33-47, 1998.

[7] M. Rucinski, D. Izzo, and F. Biscani, "On the impact of the migration topology on the island model," CoRR, vol. abs/1004.4541, 2010.

[8] Z. Skolicki and K. A. D. Jong, "The influence of migration sizes and intervals on island models," in GECCO, 2005, pp. 1295-1302.

[9] S. Gustafson and E. K. Burke, "The speciating island model: An alternative parallel evolutionary algorithm," J. Parallel Distrib. Comput., vol. 66, no. 8, pp. 1025-1036, 2006.

[10] L. Araujo, J. J. M. Guervós, A. Mora, and C. Cotta, "Genotypic differences and migration policies in an island model," in GECCO, 2009, pp. 1331-1338.

[11] F. Lardeux and A. Goëffon, "A dynamic island-based genetic algorithms framework," in SEAL, 2010, pp. 156-165.

[12] Á. Fialho, L. D. Costa, M. Schoenauer, and M. Sebag, "Extreme value based adaptive operator selection," in PPSN, 2008, pp. 175-184.

[13] B. Derbel and S. Verel, "DAMS: Distributed Adaptive Metaheuristic Selection," in Proceedings of the annual conference on Genetic and evolutionary computation (GECCO'11), United Kingdom, Jul. 2011, pp. 1-18. 